Table 1. Infection Prevention (IP) Intensive Care Unit (ICU) Multidisciplinary Team Composition

\begin{tabular}{|c|c|}
\hline Member & Role \\
\hline ID physician & Team lead and rounds facilitator; provides expert guidance on clinical application of IP and ASP concepts \\
\hline IP nurse & $\begin{array}{l}\text { Reviews patients for presence of short-term lines and devices, isolation needs, and Clostridioides difficile surveillance; ensures } \\
\text { follow up through the unit nurse champion }\end{array}$ \\
\hline ID pharmacist & Reviews patients' antimicrobial regimen for appropriateness and potential for optimization \\
\hline Unit nurse champion & Serves as a liaison for the IP team and the nursing personnel in the unit; ensures follow up of IP ICU team's recommendations \\
\hline
\end{tabular}

Note. ID, infectious diseases; ASP, antimicrobial stewardship program.

Hospital Epidemiology and Infection Control, and we collectively decided that, despite the absence of clear exposure risks, given her clinical picture and its unknown cause, testing for SARS-CoV-2 would be requested through the county public health officer. This request was first denied due to the patient's not meeting the CDC's criteria for a person under investigation (PUI), but 2 days later (on HD9) was granted by the CDC. On HD13, nasopharyngeal RT-PCR results returned positive for SARS-CoV-2. Due to ongoing critical illness, the ID consultation service made a request to the Food and Drug Administration for compassionate-use remdesivir, which was granted that same day. The first dose was administered on HD14. On HD19, the patient was extubated, and on HD31 she was discharged home.

Antimicrobial stewardship "handshake" rounds, involving the regular in-person interaction between stewardship teams and frontline providers, were first rolled out at Children's Hospital Colorado in 2013, with good results. ${ }^{3}$ Such rounds have been associated with sustained improvements in antibiotic utilization, ${ }^{4}$ high critical-care physician satisfaction, ${ }^{5}$ and improved and timely ID consultation. ${ }^{6}$ Similar IP-focused multidisciplinary teams have been shown to reduce the rate of catheter-associated urinary tract infections and central-line-associated bloodstream infections. ${ }^{7}$ However, given that this strategy is only a recent development, further research is needed to better appreciate its impact and to optimize this practice. This case highlights an additional and critical surveillance role that a multidisciplinary IP team can provide, especially in times of emerging infectious disease. Due to the identification of this case, the CDC reviewed and later revised its PUI case definition, with widespread impact on the management of the COVID-19 epidemic within the United States. ${ }^{8}$
Acknowledgments. We would like to thank Janet Peterson, Felicidad Loomis, and Shavinderpal Sanga for their roles in the care of this patient and their contribution to the multidisciplinary IP team.

Financial support. No financial support was provided relevant to this article.

Conflicts of interest. All authors report no conflicts of interest relevant to this article.

\section{References}

1. Undiagnosed pneumonia-China (Hubei). ProMED-mail website. http:// www.promedmail.org. Published December 2019. Accessed April 22, 2020.

2. Holshue M, DeBolt C, Lindquist S, et al. First case pf 2-19 novel coronavirus in the United States. N Engl J Med 2020;382:929-936.

3. Hurst A, Child J, Parker S. Intervention and acceptance rates support handshake-stewardship strategy. J Pediatric Infect Dis Soc 2019;8(2):162-165.

4. MacBrayne C, Williams M, Levek C, et al. Sustainability of handshake stewardship: extending a hand is effective years later. Clin Infect Dis 2019 Oct 4 [Epub ahead of print]. doi: 10.1093/cid/ciz650.

5. Evans B, Kosar J, Peermohamed S. Attitudes and perceptions amongst critical care physicians towards handshake antimicrobial stewardship rounds. Cureus 2019 Dec 19 [Epub ahead of print];11(12):e6419. doi: 10.7759/ cureus.6419.

6. Messacar K, Campbell K, Pearce K, Pyle L, Hurst A, Child J, Parker S. A handshake from antimicrobial stewardship opens doors for infectious disease consultation. Clin Infect Dis 2017;64:1449-1952.

7. Arora N, Patel K, Engell C, LaRosa J. The effect of interdisciplinary team rounds on urinary catheter and central venous catheter days and rates of infection. Am J Med Qual 2014;29:329-334.

8. Sanville B, Corbett R, Pidcock W, et al. A community transmitted case of severe acute respiratory distress syndrome due to SARS CoV2 in the United States. Clin Infect Dis 2020 Mar 30 [Epub ahead of print]. doi: 10. 1093/cid/ciaa347.

\title{
Surveillance for probable COVID-19 using structured data in the electronic medical record
}

\author{
Patrick C. Burke MPH, $\mathrm{CIC}^{1}$ (1), Rachel Benish Shirley $\mathrm{PhD}^{2}$, Matthew Faiman MD, MBA ${ }^{3,4}$, Eric W. Boose $\mathrm{MD}^{3,4}$, \\ Robert W. Jones Jr MD ${ }^{3}$, Amy Merlino MD ${ }^{4}$, Steven M. Gordon MD ${ }^{5}$ and Thomas G. Fraser MD ${ }^{5}$ \\ ${ }^{1}$ Department of Infection Prevention, Cleveland Clinic, Cleveland, Ohio, ${ }^{2}$ Enterprise Quality, Cleveland Clinic, Cleveland, Ohio, ${ }^{3}$ Cleveland Clinic Community \\ Care, Cleveland Clinic, Cleveland, Ohio, ${ }^{4}$ Information Technology, Cleveland Clinic, Cleveland, Ohio and ${ }^{5}$ Department of Infectious Disease, Cleveland Clinic, \\ Cleveland, Ohio
}

\footnotetext{
Author for correspondence: Patrick C. Burke, E-mail: burkep@ccf.org

Cite this article: Burke PC, et al. (2021). Surveillance for probable COVID-19 using structured data in the electronic medical record. Infection Control \& Hospital Epidemiology, 42: 781-783, https://doi.org/10.1017/ice.2020.359
}

To the Editor-Because of limited testing for COVID-19 in the community, on April 8, 2020, the Ohio Department of Health 
adopted the Council for State and Territorial Epidemiologists' (CSTE) case definition for COVID-19, ${ }^{1}$ requiring confirmed and probable cases of the disease to be reported to public health authorities within 24 hours of identification. Probable COVID-19 includes patients with compatible clinical and epidemiologic characteristics for whom no confirmatory laboratory testing has been performed and no alternative more likely diagnosis is made. Eliminating requisite laboratory confirmation for reporting cases of COVID-19 to the public health department presents a unique challenge for healthcare systems that traditionally rely on laboratory-based notification of reportable infectious diseases.

At the same time, the Cleveland Clinic was optimizing the use of our virtual encounter platforms to minimize the risk of severe acute respiratory coronavirus virus 2 (SARS-CoV-2) exposure in the traditional healthcare setting according to guidance from the Centers for Disease Control and Prevention. Virtual visits at the Cleveland Clinic are synchronous live video- and audio-enabled encounters that take place through our Express Care Online format or other commercial apps, eg, Apple FaceTime. In 2018, the Cleveland Clinic provided 33,789 virtual visits through Express Care Online. We saw the increasing demand for virtual visits in the wake of the COVID-19 pandemic as an opportunity to leverage documentation in the medical record for probable COVID-19 surveillance and reporting.

\section{Methods}

We developed a standardized clinical note template in our electronic medical record (EPIC, Epic Systems, Verona, WI) with COVID-19 Smart Data Elements (SDEs) aligned with the clinical and epidemiologic criteria in the CSTE case definition. Patient responses to these 'yes' or 'no' fields guide the healthcare provider in diagnostic and testing decision making (Supplemental Material online). Virtual visit providers at the Cleveland Clinic are prompted to use this note template when the provider selects "COVID-19 concern" as the patient's chief complaint. Patients could be referred for SARSCoV-2 testing if they were in Ohio, had a high-risk chronic condition or age, and had at least 2 of the following symptoms: fever, cough, shortness of breath, myalgia, diarrhea, anosmia, or loss of taste. SDEs from virtual visits in the structured format enable rapid daily extraction from our Enterprise Data Vault (EDV) and reporting to public health within 24 hours.

We queried the EDV for patients seen by virtual visit with at least 1 major COVID-19 criteria (ie, cough, shortness of breath, or difficulty breathing) or at least 2 minor COVID-19 criteria (ie, measured or subjective fever, chills, myalgia, headache, sore throat, new olfactory and/or taste disorder). Rigors, a minor criterion in the CSTE definition, was not included as a discrete field in our note template. We used the presence of any of the following viral infection-related International Classification of Disease, Tenth Revision (ICD-10) codes as our indication that no alternative diagnosis had been made: B34.9, viral illness; R68.89, suspected COVID-19 virus infection; Z20.828, exposure to COVID-19 virus; Z71.89, educated about COVID-19 virus infection; B97.29, other coronavirus as the cause of diseases classified elsewhere; or J22, lower respiratory infection (eg, bronchitis, pneumonia, pneumonitis, pulmonitis). We considered patient residence in the United States to satisfy the epidemiologic linkage criteria of the probable case definition, "residence in an area with sustained, ongoing community transmission of SARS-CoV-2." ${ }^{2}$ We included in our query whether the patient reported close contact with a confirmed or probable case of COVID-19.
Table 1. Clinical and Epidemiologic Characteristics of Patients Seen at the Cleveland Clinic Virtual Visit for COVID-19 Concern Who Did and Did Not Meet the CSTE Case Definition for Probable COVID-19, April 15-April 21, 2020

\begin{tabular}{|c|c|c|}
\hline Characteristic & $\begin{array}{c}\text { Probable } \\
\text { COVID-19 } \\
(\mathrm{N}=218), \\
\text { No. }(\%)\end{array}$ & $\begin{array}{l}\text { Not Probable } \\
\text { COVID-19 } \\
(N=106), \\
\text { No. }(\%)^{a}\end{array}$ \\
\hline \multicolumn{3}{|l|}{ Major clinical criteria } \\
\hline Cough & $160(74)$ & $34(32)$ \\
\hline Shortness of breath & $80(37)$ & $8(8)$ \\
\hline Difficulty breathing & $39(18)$ & $5(5)$ \\
\hline \multicolumn{3}{|l|}{ Minor clinical criteria } \\
\hline Fever (measured or subjective) & $87(40)$ & $14(13)$ \\
\hline Chills & $120(55)$ & $11(10)$ \\
\hline Rigors $^{b}$ & $\ldots$ & $\ldots$ \\
\hline Myalgia & $114(52)$ & $17(16)$ \\
\hline Headache & $119(55)$ & $30(28)$ \\
\hline Sore throat & $96(44)$ & $27(25)$ \\
\hline New olfactory and taste disorder(s) & $37(17)$ & $2(2)$ \\
\hline \multicolumn{3}{|l|}{ Epidemiologic Link } \\
\hline $\begin{array}{l}\text { Travel to or residence in an area with } \\
\text { sustained, ongoing community } \\
\text { transmission of SARS-CoV-2 }\end{array}$ & $218(100)$ & $106(100)$ \\
\hline $\begin{array}{l}\text { Close contact with confirmed or } \\
\text { probable COVID-19 }\end{array}$ & $79(36)$ & $17(16)$ \\
\hline
\end{tabular}

apatients that did not meet the probable COVID-19 case definition and no SARS-CoV-2 test

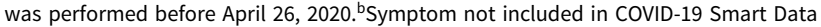
Elements.

\section{Results}

Between April 15 and April 21, 2020, 526 patients from 12 states were seen by virtual visit for COVID-19 concerns; 218 (41\%) of these met the CSTE case definition for probable COVID-19 and were reported to the public health department. Also, 167 patients who otherwise met the definition for probable COVID-19 were subsequently tested for SARS-CoV-2, so were not reported as such; $16(10 \%)$ tested positive. Of the 35 patients tested that did not meet the probable case definition due to a lack of clinical criteria or the presence of an alternative more likely diagnosis, 5 (14\%) tested positive. During the same week, the Cleveland Clinic reported 353 cases of laboratory-confirmed COVID-19 diagnosed in our hospital, emergency departments, and our drive-through testing center.

Overall, 171 patients (78\%) with probable COVID-19 reported at least 1 major clinical criteria, whereas 47 patients (22\%) reported no major criteria but 2 or more minor criteria. Furthermore, 139 patients (64\%) were classified as probable COVID-19 cases based on residence or travel in the United States, without known close contact with a confirmed or probable case of COVID-19. The clinical and epidemiologic characteristics of patients seen via virtual visits are shown in Table 1.

\section{Discussion}

Implementing structured documentation in the electronic medical record for the clinical and epidemiologic characteristics of the CSTE case definition for COVID-19 enabled us to easily find 
and report additional cases to public health authorities for investigation. Finding these previously undetected cases increased our COVID-19 reports to the public health department by $62 \%$.

The public health value added by surveillance and investigation of probable COVID-19 will become less clear as testing availability increases. Illustrating this point is our finding that, among the patients who would have been probable COVID-19 cases without being tested, fewer than $10 \%$ tested positive for SARS-CoV-2. Our findings highlight the need for more widespread testing for SARS-CoV-2 to appropriately allocate scare public health resources for COVID-19 investigation, isolation, and contact tracing.

Acknowledgments.

Financial support. No financial support was provided relevant to this article.
Conflicts of interest. All authors report no conflicts of interest relevant to this article.

Supplementary material. To view supplementary material for this article, please visit https://doi.org/10.1017/ice.2020.359

\section{References}

1. Standardized surveillance case definition and national notification for 2019 novel coronavirus disease (COVID-19). Council for State and Territorial Epidemiologists website. https://cdn.ymaws.com/www.cste.org/resource/ resmgr/2020ps/interim-20-id-01_covid-19.pdf. Published 2020. Accessed April 26, 2020.

2. Cases of coronavirus disease (COVID-19) in the US. Centers for Disease Control and Prevention website. https://www.cdc.gov/coronavirus/2019ncov/cases-updates/cases-in-us.html. Published 2020. Accessed April 26, 2020 .

\title{
Coordinated outreach for veterans in long-term care facilities by an integrated Veterans Affairs healthcare system during the COVID-19 pandemic
}

\author{
Alexander Winnett $\mathrm{BS}^{1}$ (1), Lauren P. Jatt MD ${ }^{1}$ (1), Linda Sohn MD, MPH ${ }^{1,2}$, Marcia Lysaght DNP, RN, CENP ${ }^{2}$ (1), \\ Thomas Yoshikawa MD ${ }^{1,2}$, Steven R. Simon MD, MPH ${ }^{1,2}$, Christopher J. Graber MD, MPH ${ }^{1,2}$ and \\ Matthew Bidwell Goetz MD ${ }^{1,2}$ \\ ${ }^{1}$ David Geffen School of Medicine, University of California Los Angeles, Los Angeles, California and ${ }^{2}$ Veterans Affairs Greater Los Angeles Healthcare System, \\ Los Angeles, California
}

To the Editor-We read with interest the article by Guar et al ${ }^{1}$, highlighting the burdensome and dangerous effect of the coronavirus disease 2019 (COVID-19) pandemic on long-term care facilities (LTCFs), and their recommendations to support these vulnerable populations. Even large healthcare organizations have struggled to obtain the resources necessary to maintain normal operations, ${ }^{2}$ and these challenges may be accentuated in community LTCFs with fewer staff and less financial flexibility to obtain resources for the prevention and control of severe acute respiratory coronavirus virus 2 (SARS-CoV-2) outbreaks. In response to the sentiment described by Guar et $\mathrm{al}^{1}$ that "these extraordinary times call for unprecedented measures to protect our vulnerable LTCF residents" and their recommendation for "hospital systems to include LTCF settings as high-priority sites for increased access to respiratory viral tests, including for SARS-Cov-2, and [personal protective equipment]," we offer a brief description of unprecedented measures by a large, integrated, Veterans Affairs (VA) healthcare system to support community LTCFs facing resource limitations during the COVID-19 pandemic.

The VA Greater Los Angeles Healthcare System (VAGLAHS) is a multicampus healthcare system that includes a tertiary-care

Author for correspondence: Alexander Winnett, E-mail: awinnett@mednet.ucla.edu Cite this article: Winnett A, et al. (2021). Coordinated outreach for veterans in longterm care facilities by an integrated Veterans Affairs healthcare system during the COVID-19 pandemic. Infection Control \& Hospital Epidemiology, 42: 783-784, https:// doi.org/10.1017/ice.2020.326 hospital, several satellite clinics, and on-campus LTCFs. In response to the COVID-19 outbreak amongst residents of 2 of the on-campus LTCF wards, 1 of these LTCF wards was converted to a COVID-19 recovery unit (CRU) to provide subacute medical care specifically for SARS-CoV-2-positive individuals following acute-care hospitalization, separate from uninfected LTCF residents. ${ }^{3}$ During this outbreak, VAGLAHS had access to high-volume molecular diagnostic testing capacity and utilized universal, serial, surveillance testing of residents to identify and isolate individuals at risk of transmitting the virus. These resources allowed for effective control practices ultimately halting the outbreak, with no new infections after only 2 weeks. ${ }^{4}$

However, many veterans reside in community LTCFs that are also at risk of a COVID-19 outbreak but may have more limited access to resources needed to prevent or control an outbreak, such as high-volume testing and personal protective equipment. Recognizing this dilemma, VAGLAHS leadership followed the provisions of the "Fourth Mission" of the VA to help community facilities also dealing with the pandemic: to "improve the Nation's preparedness for response to war, terrorism, national emergencies, and natural disasters by developing plans and taking actions to ensure continued service to veterans, as well as to support national, state, and local emergency management, public health, safety and homeland security efforts." ${ }^{\text {" }}$ A VAGLAHS Long-Term Care COVID-19 (LTCCV19) working group was established to take action and coordinate outreach efforts. 